\title{
DESCRIPTIVE STUDY OF EFFECT OF NARROW-BAND NOISE ON INDIVIDUALS WITH TINNITUS
}

\author{
Vinay Kumar M. V1, Beluregowda P. R², Raghavendra Prasad K. U3, Manohar S. R4, Grace Dass $A^{5}$, Rakesh P. G6
}

${ }_{1}^{1}$ Associate Professor, Department of ENT, HIMS, Hassan, Karnataka.

2 Assistant Professor, Department of ENT, HIMS, Hassan, Karnataka.

3 Professor, Department of ENT, HIMS, Hassan, Karnataka.

${ }^{4}$ Senior Resident, Department of ENT, HIMS, Hassan, Karnataka.

5Junior Resident, Department of ENT, HIMS, Hassan, Karnataka.

${ }^{6}$ Audiologist and Speech Pathologist, Department of ENT, HIMS, Hassan, Karnataka.

\section{BACKGROUND}

\section{ABSTRACT}

Residual inhibition is a temporary quieting of tinnitus that can happen after listening to the right type of sound. Studies report that more than 8 out of 10 people with tinnitus can experience at least some residual inhibition. Since there is a lack of studies done in district hospitals in India, this study has been done.

\section{MATERIALS AND METHODS}

This is a descriptive study involving patients who came with the complaints of ringing sensation (tinnitus) with reduced hearing sensitivity. All the participants were noted with detailed case history, otoscopic examination and hearing evaluation (PTA, immittance and $\mathrm{OAE}$ ) to know the degree and type of hearing loss.

\section{RESULTS}

Results were analysed in data forms. Individuals with hearing impairment who had tinnitus showed positive complete with residual inhibition. These individuals will get more benefit from hearing aids where their speech understanding abilities will be improved and the complaint of tinnitus will be masked.

\section{CONCLUSION}

Individuals with SNHL, mainly presbycusis will get more benefit from residual inhibition. These individuals will perform better with digital programmable hearing aid.

\section{KEYWORDS}

Narrow-Band Noise, Tinnitus, Screening, Hearing Aid.

HOW TO CITE THIS ARTICLE: Kumar VMV, Beluregowda PR, Prasad RKU, et al. Descriptive study of effect of narrow-band noise on individuals with tinnitus. J. Evolution Med. Dent. Sci. 2018;7(18):2243-2245, DOI: 10.14260/jemds/2018/505

\section{BACKGROUND \\ Tinnitus is one of the most common and very distressing} symptoms seen in the Dept. of ENT. There are various treatment strategies that can be used to manage tinnitus. They can be described as pharmacological treatment, surgical treatment, psychological treatment and treatment using masking. Among these treatments, using masking is most effective and non-invasive.

Tinnitus represents one of the most common and distressing otologic problems, and it causes various somatic and psychological disorders that interfere with the quality of life. Tinnitus does not represent a disease itself, but is a symptom of a variety of underlying conditions. There is considerable debate about its cause. Any pathologic lesion in the auditory pathway or any reduction in auditory nerve function has the potential to produce tinnitus. However, in many cases, no underlying physical cause is identifiable.

'Financial or Other Competing Interest': None.

Submission 12-03-2018, Peer Review 12-04-2018,

Acceptance 18-04-2018, Published 30-04-2018.

Corresponding Author:

Dr. Beluregowda P. R,

Assistant Professor,

Department of ENT,

HIMS, Hassan,

Karnataka.

E-mail: entbeluregowda@gmail.com

DOI: $10.14260 /$ jemds $/ 2018 / 505$

\section{(c) $(1) \odot$}

There are many foreign studies, which showed that with masking noise the tinnitus reduces. The purpose of this study is to evaluate the residual inhibition to determine whether the use of tinnitus maskers would be a viable treatment course. ${ }^{1}$ Residual inhibition (RI), a post-masking effect with much longer duration was first reported by Feldmann. ${ }^{2}$ The term refers to a decrease in the perceived intensity of the tinnitus for a time after the masking noise stops. Typically, residual inhibition lasts only for a few seconds or minutes, but occasionally can persist substantially longer. No concerns are reported for tests of residual inhibition in India.

\section{Objectives of the Study}

1. To compare the residual inhibition between male and female group of individuals with tinnitus.

2. To compare the residual inhibition across age groups of individuals with tinnitus.

\section{Review of Literature}

Residual inhibition refers to the phenomenon where the tinnitus perception is reduced in intensity or eliminated altogether following auditory stimulation. ${ }^{3}$ Authors discovered and developed this procedure. ${ }^{2}$ The effect has been reported to last less than 2 mins in $60 \%$ of patients and less than 4 mins in $80 \% .4$

The studies have measured post-masking effects in their patients with a one-minute masker presented at $10 \mathrm{~dB}$ above the minimum level required to mask the tinnitus. 
They reported that $90 \%$ of them demonstrated some form of residual inhibition. 5

Studies report a few cases in which 15 minutes of masking noise resulted in residual inhibition of tinnitus for the entire day. Clearly, a therapeutic technique for tinnitus that did not require continuous masking noise would be of great benefit. However, the factors resulting in persistence of inhibition and the mechanism are not well understood. 6

Authors conducted a parametric study of residual inhibition to determine the dependence of RI on masker characteristics such as centre frequency, bandwidth, intensity and duration. They wanted to know if maskers could be designed to be maximally efficient in producing RI and if the incidence and extent of RI could be predicted from the type of tinnitus. They found that RI increased with masker intensity, but the relationship to duration was not clear. Duration only increased RI when a relatively intense masker was used, and it is not known if this holds for masker durations greater than 10 minutes. The relationship of RI to tinnitus frequency and to masker center frequency and bandwidth was complex and varied between individuals. No attempt was made to assess the effect of masking noise outside the tinnitus frequency. ${ }^{7}$ In contrast, many authors they did not find evidence of contralateral masking. They found that there was no obvious predictor of whether a particular subject would exhibit RI. ${ }^{8}$

Researchers performed PET scans on 12 patients with severe, chronic tinnitus. Patients were scanned while they were experiencing their tinnitus and while their tinnitus was suppressed with either masking sounds or lidocaine injection. The results showed that cortical areas of the prefrontal and temporal lobes were active during the tinnitus situation, but not when the tinnitus was suppressed. Subtraction of the masked condition from the unmasked condition showed activity predominantly in the right hemisphere, specifically the middle frontal and middle temporal gyri as well as the lateral and medial posterior sites. These areas are linked to attention, emotion and memory. The authors hypothesised that this is why severe tinnitus becomes distressing and patients cannot habituate to it.9

\section{Source of Data}

Patients who came with the complaint of ringing sensation (Tinnitus) at HIMS, Hassan.

\section{Method of Collection Data Study Design}

This is a descriptive study proposed at Department of ENT HIMS, Hassan.

\section{Study Population}

100 individuals with the complaint of ringing or buzzing sensation (Tinnitus) with the age range of 20 to 60 years.

\section{Inclusion Criteria}

Individuals with the complaint of ringing or buzzing sensation (Tinnitus) with the age range of 20 to 60 years were taken for the study. All the participants should have the pure tone average ranging from normal-to-moderate sensory neural hearing loss will be considered for the study. All the participants must have A type tympanogram and otoscopic findings should show normal tympanic membrane to eliminate middle ear abnormality.

\section{Exclusion Criteria}

The participants should not have any history of neurologic or otologic disorders. None of the participants should use any amplification devices (Hearing aids) or tinnitus maskers. The individuals with profound hearing loss will be excluded from the study. None of the participants should have undergone tinnitus management or therapy.

\section{MATERIALS AND METHODS}

Audiometer, Immittance, Otoscope, TDH 49 Headphones.

\section{Instrumentation}

A calibrated diagnostic Elkon audiometer with Telephonics TDH-49 supra-aural headphones and Radioear B-71 bone vibrator was used for threshold estimation and the frequency of tinnitus can be obtained.

\section{Procedure}

A detailed case history will be taken before the commencement of routine audiological assessment. Puretone thresholds will be obtained at octave frequencies between $250 \mathrm{~Hz}$ to $8000 \mathrm{~Hz}$ for air conduction and between $250 \mathrm{~Hz}$ to $4000 \mathrm{~Hz}$ for bone conduction.

Individuals with tinnitus will be assessed and a detail tinnitus evaluation will be done. The narrow-band noise will be presented from Elkon audiometer via calibrated Telephonics TDH 49 headphones for 1 minute. Tests are performed through headphones. The most effective masking signal is presented to the ipsilateral ear at $10 \mathrm{~dB}$ above the minimal masking sensation level (where possible) for 60 seconds. Then the post-masking effects are classified into four categories.

\section{These Categories are-}

1. Positive-complete, where the tinnitus is completely absent for more than one minute;

2. Positive-partial, where the tinnitus is still present but softer at a lower perceived level than before for more than one minute;

3. Negative, where there is no reported change in the tinnitus; and finally,

4. Rebound, where the tinnitus is actually louder after the masking stimulus is presented. Patients who fall under 1 and 2 will get more benefit from tinnitus maskers.

\section{RESULTS}

100 individuals with the complaint of ringing or buzzing sensation (tinnitus) with the age range of 20 to 60 years were taken for the study. Among them, 43 were males and 57 were females. Most of the individuals were reported positive residual inhibition effect.

\section{Among 100 Individuals-}

- 62 fell under Positive-complete, where the tinnitus was completely absent for more than one minute.

- 22 were in Positive-partial, where the tinnitus was still present, but softer at a lower perceived level than before for more than one minute; 
- 14 were in Negative, where there was no reported change in the tinnitus; and finally

- 2 were in Rebound, where the tinnitus was actually louder after the masking stimulus was presented.

\begin{tabular}{|c|c|c|c|c|}
\hline Gender & $\begin{array}{c}\text { Positive } \\
\text { Complete }\end{array}$ & $\begin{array}{c}\text { Positive- } \\
\text { Partial }\end{array}$ & Negative & Rebound \\
\hline Males & 28 & 8 & 6 & 1 \\
\hline Females & 34 & 14 & 8 & 1 \\
\hline \multicolumn{5}{|c|}{ Table 1 } \\
\hline
\end{tabular}

In our study, 43 males and 57 females were taken. Among 43 males 28 showed positive complete, 8 showed positive partial, 6 were negative and 1 rebound. Among 57 females 34 showed positive complete, 14 showed positive partial, 8 were negative and 1 rebound. This shows among 100 individuals irrespective of gender, 84 individuals will get benefit from tinnitus maskers and digital hearing aids.

\begin{tabular}{|c|c|c|c|c|}
\hline $\begin{array}{c}\text { Age } \\
\text { Groups }\end{array}$ & $\begin{array}{c}\text { Positive } \\
\text { Complete }\end{array}$ & $\begin{array}{c}\text { Positive } \\
\text { Partial }\end{array}$ & Negative & Rebound \\
\hline $20-30$ & 1 & - & - & - \\
\hline $30-40$ & 4 & 2 & - & - \\
\hline $40-50$ & 35 & 11 & 6 & 1 \\
\hline $50-60$ & 22 & 9 & 8 & 1 \\
\hline \multicolumn{5}{|c|}{ Table 2 } \\
\hline
\end{tabular}

In our study, age groups from 20 to 60 years were distributed in four sub-groups.

The age group of 20 to 40 had only 7 participants, among them 5 showed positive-complete and 2 fell under positivepartial. Under this age group, none of them showed Negative or Rebound results.

In the age group of 40 to 50 we had 53 participants, among them 35 showed Positive-complete, 11 fell under Positive-partial, 6 were negative and 1 rebound.

In the age group of 50 to 60 we had 40 participants, among them 22 showed Positive-complete, 9 fell under Positive-partial, 8 were negative and 1 rebound.

Those individuals who showed Positive-complete and Positive-partial were likely to be benefitted well with tinnitus maskers and digital hearing aids.

\section{DISCUSSION}

Tinnitus is one of the most common problems seen in ENT OPD, usually self-limiting. But in some patients, it will be a very distressing symptom. Many treatment options are available which include both medical and surgical treatments. If the patients opts for surgical treatment, they may have to compromise with their hearing abilities. For this reason, tinnitus maskers are available or the digital hearing aids which will be helpful for those individuals who has sensorineural hearing loss with tinnitus. In our study it revealed that among 100 individuals with tinnitus, 84 of them will get better benefit from hearing aids and maskers. Those individuals who showed negative and rebound tinnitus are not the best candidates for tinnitus maskers, but they can get rehabilitation by using digital programmable hearing aids to restore hearing abilities. Those individuals who showed Positive-complete and Positive-partial were likely to be benefitted well with tinnitus maskers and digital hearing aids.

\section{CONCLUSION}

Patients who fall under the categories 1 and 2 will get more benefit from tinnitus maskers. Due to decrease in hearing, there is more activity happening in brainstem to cochlea. That in turn leads to tinnitus with sufficient amount of noise to the inner ear. The individuals who are having tinnitus will not be able to hear the reverse signal that is tinnitus. So, individuals with SNHL, mainly presbycusis, will get more benefit from residual inhibition. These individuals will perform better with digital programmable hearing aid.

In individuals who were classified in rebound category will not get any benefit from maskers of residual inhibition. This may be due to those individuals who might have retrocochlear pathology in them along with cochlear pathology.

\section{REFERENCES}

[1] Shulman A, Strashun AM, Seibyl JP, et al. Benzodiazepine receptor deficiency and tinnitus. Int Tinnitus J 2000;6(2):98-111.

[2] Feldmann H. Homolateral and contralateral masking of tinnitus by noise bands and pure tones. Audiology 1971;10(3):138-44.

[3] Vernon JA. Some observations on residual inhibition. In: Paparella MM, Meyerhoff WL. (eds). Sensorineural hearing loss, vertigo and tinnitus. Ear Clinics International. Baltimore: Williams \& Wilkins, 1981:13844.

[4] Meikle MB, Creedon TA, Griest SE. Tinnitus archive. $2^{\text {nd }}$ edn. Oregon hearing research center, Department of Otolaryngology. Oregon Health \& Science University, 2004.

[5] Vernon JA, Meikle MB. Tinnitus masking: unresolved problems. Ciba Found Symp 1981;85:239-62.

[6] Vernon JA, Meikle MB. Tinnitus masking: unresolved problems. In Evered D, Lawrenson G. (Eds). Ciba Foundation Symposium 85-Tinnitus, John Wiley \& Sons, Chichester, UK, 1981:239-62.

[7] Hazell JW, Wood S. Tinnitus masking-a significant contribution to tinnitus management. $\mathrm{Br} \mathrm{J}$ Audiol 1981;15(4):223-30.

[8] Terry AM, Jones DM, Davis BR, et al. Parametric studies of tinnitus masking and residual inhibition. Br J Audiol 1983;17(4):245-56.

[9] Mirz F, Pedersen B, Ishizu K, et al. Positron emission tomography of cortical centers of tinnitus. Hear Res 1999;134(1-2):133-44. 